\title{
DETERMINANTS OF NORMALIZED BOHEMIAN UPPER HESSENBERG MATRICES*
}

\author{
MASSIMILIANO FASI ${ }^{\dagger}$ AND GIAN MARIA NEGRI PORZIO P $^{\ddagger}$
}

\begin{abstract}
A matrix is Bohemian if its elements are taken from a finite set of integers. A upper Hessenberg matrix is normalized if all its subdiagonal elements are ones, and hollow if it has only zeros along the main diagonal. All possible determinants of families of normalized and hollow normalized Bohemian upper Hessenberg matrices are enumerated. It is shown that in the case of hollow matrices the maximal determinants are related to a generalization of Fibonacci numbers. Several conjectures recently stated by Corless and Thornton follow from these results.
\end{abstract}

Key words. Bohemian matrix, Integer matrix, Normalized upper Hessenberg matrix, Determinant, Fibonacci number.

AMS subject classifications. 11C20, 15A15, 15B36.

1. Introduction. Matrices whose entries are drawn from a finite discrete set $\mathcal{D}$ are said to be Bohemian. The term is a partial acronym for "BOunded HEight Matrix of Integers", and is due to the fact that $\mathcal{D}$ is bounded, being finite, and is typically a subset of the integers. This terminology is of recent introduction [5], but Bohemian matrices have been a subject of interest for at least a century and a half, with early work of Sylvester [10] and Hadamard [9] dating back to the second half of the nineteenth century.

The study of families of matrices with integer elements flourished in the sixties: multiple authors examined theoretical [11] as well as practical [7] applications of Bohemian matrices, and many important conjectures on the subject were first stated in that decade. A good example is the expository survey of "computational problems involving integral matrices" collected by Taussky [12], where combinatorial problems involving matrices of integers are tackled, for small dimensions, by means of a brute force, computer-aided approach.

More recently, Chan et al. studied the distribution of the eigenvalues and the height of the characteristic polynomials of families of Bohemian upper Hessenberg [3] and upper Hessenberg Toeplitz matrices [2]. Many observations made by the authors while preparing these two manuscripts were collected in the form of conjectures in the Characteristic Polynomial Database [13].

Some of these conjectures have been either proved or disproved since, but many remain open. Here we focus on those that state properties of the determinants of families of normalized upper Hessenberg matrices, that is, upper Hessenberg matrices with ones on the subdiagonal and elements in the upper triangular part drawn from the sets $\{0,1\},\{0,-1\},\{-1,1\},\{-1,0,1\}$, and $\{0,1,2\}$. Our goal will be to enumerate all possible determinants for matrices with these structures: this will allow us to count the number of distinct determinants and find the maximum absolute determinant of each of these matrix families. We will often

${ }^{*}$ Received by the editors on May 12, 2019. Accepted for publication on March 18, 2020. Handling Editor: Bryan L. Shader. Corresponding Author: Massimiliano Fasi.

$\dagger$ Department of Mathematics, University of Manchester, M13 9PL, UK (massimiliano.fasi@manchester.ac.uk). The work of this author was supported by MathWorks, the Royal Society, and the Istituto Nazionale di Alta Matematica, INdAM-GNCS Project 2019. The opinions and views expressed in this publication are those of the author, and not necessarily those of the funding bodies.

¥Department of Mathematics, University of Manchester, M13 9PL, UK (gianmaria.negriporzio@manchester.ac.uk). 
be able to work with more general domains, thus proving the aforementioned conjectures as special cases.

The next section introduces our notation and recalls background definitions and results that will be used later on. In Sections 3 and 4, we discuss the determinants of families of normalized and normalized hollow Bohemian upper Hessenberg matrices, respectively, and prove a generalization of most of the open conjectures in the Characteristic Polynomial Database. Finally, in Section 5, we prove [13, Conjecture 8], the only conjecture we were not able to prove in its generalized form, and state our generalization.

2. Background and notation. Here and in the following sections, $n$ and $d$ always denote positive integers. We say that the matrix $H \in \mathbb{C}^{n \times n}$ is upper Hessenberg if $h_{i j}=0$ for $i>j+1$. An upper Hessenberg matrix $H \in \mathbb{C}^{n \times n}$ is normalized if $h_{i+1, i}=1$, for $i=1, \ldots, n-1$, and is hollow if $h_{i i}=0$, for $i=1, \ldots, n$. For any $\mathcal{D} \subset \mathbb{Z}$, we denote by $\mathcal{H}^{n}(\mathcal{D})$ the family of normalized upper Hessenberg matrices with elements from $\mathcal{D}$ in the upper triangular part, and by $\mathcal{H}_{0}^{n}(\mathcal{D})$ the family of hollow normalized upper Hessenberg matrices with elements from $\mathcal{D}$ in the strictly upper triangular part.

It is useful to introduce a shorthand notation for integer intervals. For any $i_{1}, i_{2} \in \mathbb{Z}$ such that $i_{1}<i_{2}$ we denote by $\left\langle i_{1}, i_{2}\right\rangle$ the set of integers between $i_{1}$ and $i_{2}$ inclusive, and if $i_{1}<0<i_{2}$ we denote by $\left\langle i_{1}, i_{2}\right\rangle^{\circ}$ the set $\left\langle i_{1}, i_{2}\right\rangle \backslash\{0\}$.

Finally, we recall a well-known result that can be used to write the determinant of a normalized upper Hessenberg matrix in terms of its leading principal minors.

Lemma 2.1. ([6, Section 7.11]) Let $H \in \mathcal{H}^{n}(\mathcal{D})$. Then

$$
\operatorname{det} H=(-1)^{n+1}\left(h_{1 n}+\sum_{i=2}^{n}(-1)^{i-1} h_{i n} \operatorname{det} H^{(i-1)}\right)
$$

where $H^{(i)} \in \mathcal{H}^{i}(\mathcal{D})$ is the ith leading principal submatrix of $H$.

Proof. The identity can be verified by expanding along the last column of $H$.

3. Normalized Bohemian upper Hessenberg matrices. In this section, we consider normalized Bohemian upper Hessenberg matrices with elements in the upper triangular part drawn from $\langle-d, d\rangle,\langle-d, 0\rangle$, and $\langle-d, d\rangle^{\circ}$. First we will find a closed expression for the maximum absolute value of the determinant of matrices in these classes, then we will show how to build matrices with a given determinant.

Proposition 3.1. We have that

$$
\max _{H \in \mathcal{H}^{n}(\langle-d, d\rangle)}|\operatorname{det} H|=\max _{H \in \mathcal{H}^{n}(\langle-d, 0\rangle)}|\operatorname{det} H|=\max _{H \in \mathcal{H}^{n}\left(\langle-d, d\rangle^{\circ}\right)}|\operatorname{det} H|=d(d+1)^{n-1},
$$

where the maximum is attained by the matrix $K^{(d, n)}$, defined by

$$
k_{i j}^{(d, n)}= \begin{cases}0, & i>j+1, \\ 1, & i=j+1, \\ -d, & i \leq j\end{cases}
$$

for which

$$
\operatorname{det} K^{(d, n)}=(-1)^{n} d(d+1)^{n-1} .
$$


Proof. We begin by showing (3.2). It is easy to verify that the identity holds for $n=1$. For the inductive step, from Lemma 2.1 we have that

$$
\begin{aligned}
\operatorname{det} K^{(d, n)} & =(-1)^{n+1}\left(k_{1 n}^{(d, n)}+\sum_{i=2}^{n}(-1)^{i-1} k_{i n}^{(d, n)} \operatorname{det} K^{(d, i-1)}\right) \\
& =(-1)^{n}\left(d+\sum_{i=2}^{n} d^{2}(d+1)^{i-2}\right) \\
& =(-1)^{n}\left(d+d^{2} \frac{1-(d+1)^{n-1}}{1-(d+1)}\right) \\
& =(-1)^{n} d(d+1)^{n-1} .
\end{aligned}
$$

Using the notation in Lemma 2.1 gives, for any $H \in \mathcal{H}^{n}(\langle-d, d\rangle)$,

$$
\begin{aligned}
|\operatorname{det} H| & =\left|h_{1 n}+\sum_{i=2}^{n}(-1)^{i-1} h_{i n} \operatorname{det} H^{(i-1)}\right| \\
& \leq d+\sum_{i=2}^{n} d^{2}(d+1)^{i-2} \\
& =\left|\operatorname{det} K^{(d, n)}\right|,
\end{aligned}
$$

where the last equality follows from (3.3). Observing that $K^{(d, n)}$ belongs to $\mathcal{H}^{n}(\langle-d, 0\rangle)$ and $\mathcal{H}^{n}\left(\langle-d, d\rangle^{\circ}\right)$ and that both sets are subset of $\mathcal{H}^{n}(\langle-d, d\rangle)$ concludes the proof.

Note that Proposition 3.1 generalizes [13, Conjecture 4], since for $d=1$ it shows that the sequence of maximal absolute determinants of normalized upper Hessenberg matrices with entries from the set $\langle-1,1\rangle$ is given by the OEIS sequence A03433.

Next we prove three technical results that will be necessary in order to construct a Bohemian matrix with a given determinant.

Lemma 3.2. For any $\gamma \in\left\langle 0, d(d+1)^{n-1}\right\rangle$, there exist $\beta, \alpha_{0}, \ldots, \alpha_{n-2} \in\langle 0, d\rangle$ such that

$$
\gamma=\beta+d \sum_{i=0}^{n-2} \alpha_{i}(d+1)^{i} .
$$

Proof. If $\gamma$ is strictly smaller than $d(d+1)^{n-1}$, then there exists a unique pair of integers $\mu \in\langle 0, d-1\rangle$ and $\nu \in\left\langle 0,(d+1)^{n-1}-1\right\rangle$ such that $\gamma=\mu+d \nu$. Since $\nu \leq(d+1)^{n-1}-1$, we can write

$$
\nu=\sum_{i=0}^{n-2} \alpha_{i}(d+1)^{i}
$$

and the representation (3.4) is obtained by setting $\beta$ to $\mu$ and $\alpha_{0}, \ldots, \alpha_{n-2} \in\langle 0, d\rangle$ to the $n-1$ digits in the representation of $\nu$ in radix $d+1$. If $\gamma=d(d+1)^{n-1}$, on the other hand, setting $\beta, \alpha_{0}, \ldots, \alpha_{n-2}$ to $d$ gives

$$
d+d \sum_{i=0}^{n-2}(d+1)^{i}=d+d\left((d+1)^{n-1}-1\right)=d(d+1)^{n-1},
$$

which concludes the proof. 


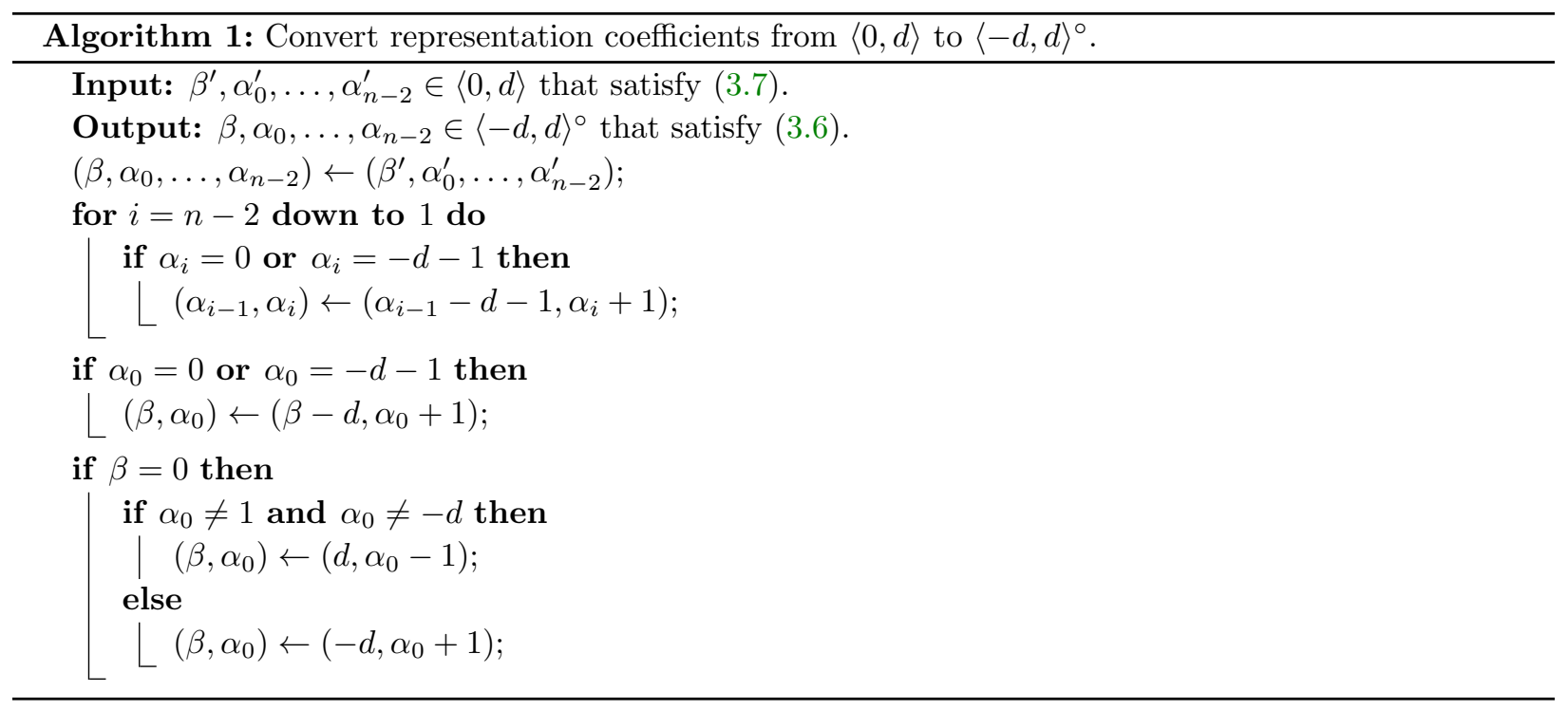

Corollary 3.3. For any $\gamma \in\left\langle-d(d+1)^{n-1}, d(d+1)^{n-1}\right\rangle$, there exist $\beta, \alpha_{0}, \ldots, \alpha_{n-2} \in\langle-d, d\rangle$ such that

$$
\gamma=\beta+d \sum_{i=0}^{n-2} \alpha_{i}(d+1)^{i}
$$

Proof. If $\gamma$ is nonnegative, then the result follows from Lemma 3.2. Otherwise, $\beta, \alpha_{0}, \ldots, \alpha_{n-2}$ can be obtained by changing the sign of the corresponding coefficients in the representation (3.4) for $-\gamma$.

A consequence of Lemma 3.2 and Corollary 3.3 is that all integers in the intervals $\left\langle 0, d(d+1)^{n-1}\right\rangle$ and $\left\langle-d(d+1)^{n-1}, d(d+1)^{n-1}\right\rangle$ have at least one representation of the form (3.4) and (3.5), respectively. In the remainder of this section, we will obtain determinantal formulae of the forms (3.4) and (3.5) and will exploit the representation results above to construct Bohemian matrices with a given determinant.

The next result shows that the representation (3.5) can be rewritten so to have only nonzero coefficients.

LEMMA 3.4. If $d>1$, for any $\gamma \in\left\langle-d(d+1)^{n-1}, d(d+1)^{n-1}\right\rangle$, there exist $\beta, \alpha_{0}, \ldots, \alpha_{n-2} \in\langle-d, d\rangle^{\circ}$ such that

$$
\gamma=\beta+d \sum_{i=0}^{n-2} \alpha_{i}(d+1)^{i}
$$

Proof. If $\gamma \geq 0$, then by Lemma 3.2, there exist $\beta^{\prime}, \alpha_{0}^{\prime}, \ldots, \alpha_{n-2}^{\prime}$ in $\langle 0, d\rangle$ such that

$$
\gamma=\beta^{\prime}+d \sum_{i=0}^{n-2} \alpha_{i}^{\prime}(d+1)^{i} .
$$

Algorithm 1 shows how the coefficients of the representation (3.6) can be computed from those of (3.7). In order to prove the correctness of the algorithm, note that the two sets of coefficients represent the same number, that all zero coefficients in (3.7) are changed into a positive or negative number smaller than 
$d$ in absolute value, and that no new zero coefficients are introduced. If $\gamma<0$, it suffices to find the representation (3.7) for $-\gamma$ in $\langle 0, d\rangle$ and change the sign of its coefficients.

The result in Lemma 3.4 is not true for $d=1$. In order to represent $n=3$ in the form (3.6), for instance, we would have to find $\beta, \alpha_{0}$, and $\alpha_{1}$ in $\{-1,1\}$ such that $\beta+\alpha_{0}+2 \alpha_{1}=3$. But such a triple cannot exist: the right-hand side is odd, but the left-hand side is even, being the sum of the two even numbers $\beta+\alpha_{0}$ and $2 \alpha_{1}$. Therefore, we will need two different strategies to prove the result for $d \neq 1$ and $d=1$.

Proposition 3.5. If $d>1$, then the set of possible determinants of matrices in the family $\mathcal{H}^{n}\left(\langle-d, d\rangle^{\circ}\right)$ is $\left\langle-d(d+1)^{n-1}, d(d+1)^{n-1}\right\rangle$.

Proof. In this case, we show how to construct a matrix $H \in \mathcal{H}^{n}\left(\langle-d, d\rangle^{\circ}\right)$ such that $\operatorname{det} H=k$ for any $k \in\left\langle-d(d+1)^{n-1}, d(d+1)^{n-1}\right\rangle$. Here we take the matrix

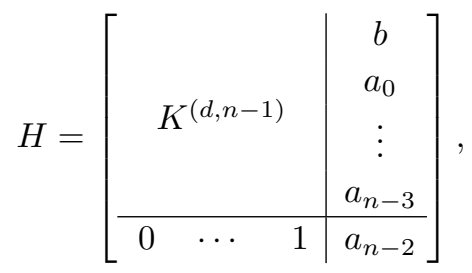

where $K^{(d, n-1)}$ is defined in (3.1). From Lemma 2.1 and Proposition 3.1, it follows that

$$
\begin{aligned}
\operatorname{det} H & =(-1)^{n+1} b+\sum_{i=0}^{n-2}(-1)^{n+i} a_{i} \operatorname{det} K^{(d, i+1)} \\
& =\widetilde{b}+d \sum_{i=0}^{n-2} \widetilde{a}_{i}(d+1)^{i}
\end{aligned}
$$

where $\widetilde{b}=(-1)^{n+1} b$ and $\widetilde{a}_{i}=(-1)^{n+1} a_{i}$, for $i=0, \ldots, n-2$. The coefficients $b, a_{0}, \ldots, a_{n-2}$ belong to $\langle-d, d\rangle^{\circ}$, and in view of Lemma 3.4 the last column of $H$ can be chosen so that $\operatorname{det} H=k$ for any $k \in\left\langle-d(d+1)^{n-1}, d(d+1)^{n-1}\right\rangle$.

Proposition 3.6. The set of possible determinants of matrices in the family $\mathcal{H}^{n}\left(\langle-1,1\rangle^{\circ}\right)$ is

$$
\{2 k \mid k \in\langle-n+1, n-1\rangle\}
$$

Proof. As noted by Ching [4], there are only $2^{n-1}$ possibly nonzero terms in the determinant expansion of an $n \times n$ Hessenberg matrix. If the matrix is in $\mathcal{H}^{n}\left(\langle-1,1\rangle^{\circ}\right)$, then each of these $2^{n-1}$ monomials evaluates to either +1 or -1 , which implies that the determinant of any such matrices must be even and cannot be larger than $2^{n-1}$ in absolute value. Now we explain how to construct a matrix $H \in \mathcal{H}_{0}^{n}\left(\langle-1,1\rangle^{\circ}\right)$ such that $\operatorname{det} H=2 k$ for any $k \in\langle 0, n-1\rangle$. Matrices with negative determinants can be obtained by changing the sign of the last column of the matrices thus obtained.

Let us consider the matrix

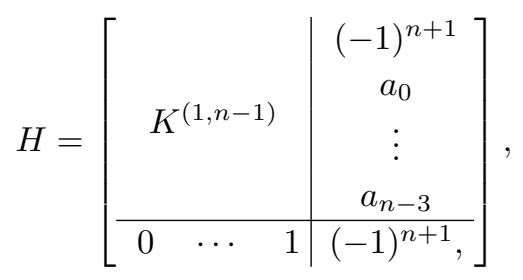


where $K^{(1, n-1)}$ is defined in (3.1). Using Lemma 2.1 followed by Proposition 3.1, we obtain that

$$
\begin{aligned}
\operatorname{det} H & =1+\sum_{i=0}^{n-3}(-1)^{n+1} a_{i} \operatorname{det} K^{(1, i+1)}+(-1)^{n+1} \operatorname{det} K^{(1, n-1)} \\
& =1+(-1)^{n+1} \sum_{i=0}^{n-3} a_{i} 2^{i}+2^{n-2}
\end{aligned}
$$

Let $k^{\prime}:=2^{n-2}-k$ and let $b_{0}, \ldots, b_{n-3} \in\{0,1\}$ be such that

$$
k^{\prime}=\sum_{i=0}^{n-3} b_{i} 2^{i}=\sum_{i \in I} b_{i} 2^{i}
$$

where $I:=\left\{i \in \mathbb{N}: b_{i} \neq 0\right\}$. If we set $a_{i}=(-1)^{n+1+b_{i}}$, we are taking the coefficients that would create the matrix $K^{(d, n)}$ with the last column multiplied by $(-1)^{n+1}$ and changing the signs in the corresponding nonzero coefficients of the binary representation of $k^{\prime}$. It follows that

$$
\begin{aligned}
\operatorname{det} H & =1+(-1)^{b_{0}}+\cdots+(-1)^{b_{n-3}} 2^{n-3}+2^{n-2} \\
& =1+\sum_{i=0}^{n-2} 2^{i}-2 \sum_{i \in I} b_{i} 2^{i} \\
& =2^{n-1}-2 k^{\prime}=2 k,
\end{aligned}
$$

which concludes the proof.

Proposition 3.7. For any $d \in \mathbb{N}$, the set of possible determinants of matrices in the family $\mathcal{H}^{n}(\langle-d, d\rangle)$ is $\left\langle-d(d+1)^{n-1}, d(d+1)^{n-1}\right\rangle$.

Proof. The proof is analogous to that of Proposition 3.5, if Lemma 3.4 is replaced by Corollary 3.3.

Proposition 3.8. For any $d \in \mathbb{N}$, the set of possible determinants of matrices in the family $\mathcal{H}^{n}(\langle-d, 0\rangle)$ is $\left\{(-1)^{n} k \mid k \in\left\langle 0, d(d+1)^{n-1}\right\rangle\right\}$.

Proof. This proof follows the steps of the proof of Proposition 3.5. Note that $\mathcal{H}^{n}(\langle-d, 0\rangle) \subset \mathcal{H}^{n}(\langle-d, d\rangle)$, and that since $K^{(d, n-1)} \in \mathcal{H}^{n}(\langle-d, 0\rangle)$, the matrix $H$ in $(3.8)$ is in $\mathcal{H}^{n}(\langle-d, 0\rangle)$ if $b, a_{0}, \ldots, a_{n-2} \in\langle-d, 0\rangle$. Combining Lemma 2.1 and Proposition 3.1 in this case gives

$$
\begin{aligned}
\operatorname{det} H & =(-1)^{n+1} b+\sum_{i=0}^{n-2}(-1)^{n+i} a_{i} \operatorname{det} K^{(d, i+1)} \\
& =(-1)^{n}\left(\widetilde{b}+d \sum_{i=0}^{n-2} \widetilde{a}_{i}(d+1)^{i}\right)
\end{aligned}
$$

where $\widetilde{b}=-b$ and $\widetilde{a}_{i}=-a_{i}$, for $i=0, \ldots, n-2$. Since $\widetilde{b}, \widetilde{a}_{0}, \ldots, \widetilde{a}_{n-2} \in\langle 0, d\rangle$, by Lemma 3.2 we can choose the last column of $H$ so that det $H=(-1)^{n} k$ for all $k \in\left\langle 0, d(d+1)^{n-1}\right\rangle$.

Note that Propositions 3.8, 3.7, and 3.6 prove [13, Conjecture 18], [13, Conjecture 19], and [13, Conjecture 20], respectively: they show that the number of distinct determinants of normalized Bohemian upper Hessenberg matrices with entries from the sets $\langle-1,0\rangle,\langle-1,1\rangle$, and $\langle-1,1\rangle^{\circ}$ is given by a suitable shift of the OEIS sequence A000051. 
4. Hollow normalized Bohemian upper Hessenberg matrices. In the previous section, we explained how to build a Bohemian upper Hessenberg matrix for every possible determinant, and by doing so we generalized some of the conjectures in [13]. Now, we follow the same steps and prove the corresponding conjectures for the normalized hollow Bohemian upper Hessenberg matrices in the family $\mathcal{H}_{0}^{n}(\mathcal{D})$ for the same finite domains as in the previous section.

We will rely on the sequence of $d$-weighted Fibonacci numbers, which are generated by the two-term recurrence

$$
\left\{\begin{array}{l}
f_{0}^{d}=0, \\
f_{1}^{d}=d, \\
f_{n}^{d}=f_{n-1}^{d}+d f_{n-2}^{d}, \quad n \in \mathbb{N} \backslash\{0,1\} .
\end{array}\right.
$$

Note that for $d=1$ one obtains the sequence of Fibonacci numbers. In that case we drop the superscript and write $f_{n}:=f_{n}^{1}$ to indicate the term in position $n$ in the OEIS sequence A000045.

The next lemma shows one possible way of constructing a matrix with maximum absolute determinant for all families of interest in this section.

Proposition 4.1. We have that

$$
\max _{H \in \mathcal{H}_{0}^{n}(\langle-d, d\rangle)}|\operatorname{det} H|=\max _{H \in \mathcal{H}_{0}^{n}(\langle-d, 0\rangle)}|\operatorname{det} H|=\max _{H \in \mathcal{H}_{0}^{n}\left(\langle-d, d\rangle^{\circ}\right)}|\operatorname{det} H|=f_{n-1}^{d},
$$

where the maximum is attained by the matrix $\widehat{K}^{(d, n)}$, defined by

$$
k_{i j}^{(d, n)}= \begin{cases}0, & i>j+1 \text { or } i=j, \\ 1, & i=j+1, \\ -d, & i<j\end{cases}
$$

for which

$$
\operatorname{det} \widehat{K}^{(d, n)}=(-1)^{n} f_{n-1}^{d}
$$

Proof. First we prove (4.12) by induction. For the base case, it is easy to verify that that $\operatorname{det} \widehat{K}^{(d, 1)}=0$ and $\operatorname{det} \widehat{K}^{(d, 2)}=d$. For the inductive step, by subtracting the $i$ th row from the row above it, for $i$ from 2 to $n$, we obtain

$$
\operatorname{det} \widehat{K}^{(d, n)}=\left|\begin{array}{ccccc}
0 & -d & -d & \cdots & -d \\
1 & 0 & -d & \cdots & -d \\
& 1 & \ddots & \ddots & \vdots \\
& & \ddots & 0 & -d \\
& & & 1 & 0
\end{array}\right|=\left|\begin{array}{ccccc}
-1 & -d & & & \\
1 & -1 & -d & & \\
& 1 & \ddots & \ddots & \\
& & \ddots & -1 & -d \\
& & & 1 & 0
\end{array}\right| .
$$

By using the Laplace expansion by minors along the first column of the tridiagonal matrix thus obtained, 
we get

$$
\operatorname{det} \widehat{K}^{(d, n)}=-\operatorname{det} \widehat{K}^{(d, n-1)}-\left|\begin{array}{ccccc}
-d & & & & \\
1 & -1 & -d & & \\
& 1 & \ddots & \ddots & \\
& & \ddots & -1 & -d \\
& & & 1 & 0
\end{array}\right|
$$

and by expanding along the first row, we can conclude that

$$
\begin{aligned}
\operatorname{det} \widehat{K}^{(d, n)} & =-\operatorname{det} \widehat{K}^{(d, n-1)}+d \operatorname{det} \widehat{K}^{(d, n-2)} \\
& =(-1)^{n} f_{n-2}^{d}+(-1)^{n-2} d f_{n-3}^{d}=(-1)^{n} f_{n-1}^{d} .
\end{aligned}
$$

In order to prove the optimality result in (4.10), we define the family of Bohemian Hessenberg matrices $\mathcal{B}^{n}=\left\{H+x e_{n} e_{n}^{T}: H \in \mathcal{H}_{0}^{n}(\langle-d, d\rangle), x \in\langle-d, d\rangle\right\}$, and show by induction that for all $H \in \mathcal{H}_{0}^{n}(\langle-d, d\rangle)$ and all $B \in \mathcal{B}^{n}$, we have that $|\operatorname{det} H| \leq f_{n-1}^{d}$ and $|\operatorname{det} B| \leq f_{n}^{d}$.

The only matrix in $\mathcal{H}_{0}^{1}(\langle-d, d\rangle)$ has determinant 0 , and it is easy to check by exhaustion that the absolute value of the determinant of matrices in $\mathcal{H}_{0}^{2}(\langle-d, d\rangle), \mathcal{B}^{1}$, and $\mathcal{B}^{2}$ is at most $d$. For the inductive step, let us consider the matrices

$$
H=\left[\begin{array}{ccccc}
0 & \times & \times & \cdots & \times \\
1 & 0 & \times & \cdots & \times \\
& 1 & \ddots & \ddots & \vdots \\
& & \ddots & 0 & \times \\
& & 1 & 0
\end{array}\right] \in \mathcal{H}_{0}^{n}(\langle-d, d\rangle) \text { and } B=\left[\begin{array}{ccccc}
0 & \times & \times & \cdots & \times \\
1 & 0 & \times & \cdots & \times \\
& 1 & \ddots & \ddots & \vdots \\
& & \ddots & 0 & \times \\
& & & 1 & b_{n n}
\end{array}\right] \in \mathcal{B}^{n}
$$

For the first matrix, we have that $|\operatorname{det} H|=\left|-\operatorname{det} B^{\prime}\right|$ for some $B^{\prime} \in \mathcal{B}^{n-1}$, and by the inductive hypothesis we can conclude that $|\operatorname{det} H| \leq f_{n-1}^{d}$. For the matrix $B$, by observing that $\operatorname{det} B=b_{n n} \operatorname{det} H^{\prime}-\operatorname{det} B^{\prime}$, for some $H^{\prime} \in \mathcal{H}_{0}^{n-1}(\langle-d, d\rangle)$ and $B^{\prime} \in \mathcal{B}^{n-1}$, we obtain that

$$
|\operatorname{det} B|=\left|b_{n n} \operatorname{det} H^{\prime}-\operatorname{det} B^{\prime}\right| \leq\left|b_{n n}\right|\left|\operatorname{det} H^{\prime}\right|+\left|\operatorname{det} B^{\prime}\right| \leq d f_{n-2}^{d}+f_{n-1}^{d}=f_{n}^{d} .
$$

We conclude that the absolute value of the determinant of matrices in the family $\mathcal{H}_{0}^{n}(\langle-d, d\rangle)$ is bounded by $f_{n-1}^{d}$, and observing that $\widehat{K}^{(d, n)}$ belongs to $\mathcal{H}_{0}^{n}(\langle-d, d\rangle)$ shows that the bound is attained by a matrix in that family. The optimality result in (4.10) follows from the fact that $\mathcal{H}_{0}^{n}(\langle-d, 0\rangle)$ and $\mathcal{H}_{0}^{n}\left(\langle-d, d\rangle^{\circ}\right)$ are subfamilies of $\mathcal{H}_{0}^{n}(\langle-d, d\rangle)$ and that $\widehat{K}^{(d, n)}$ belongs to $\mathcal{H}_{0}^{n}(\langle-d, 0\rangle) \cap \mathcal{H}_{0}^{n}\left(\langle-d, d\rangle^{\circ}\right)$.

For $d=1$, the three equalities in (4.10) prove [13, Conjecture 13], [13, Conjecture 15], and [13, Conjecture 17]. We stress that $\widehat{K}^{(d, n)}$ is not the only matrix $H \in \mathcal{H}_{0}^{n}(\langle-d, d\rangle)$ such that $|\operatorname{det} H|=f_{n-1}^{d}$.

Now we can use these results to build matrices with specific determinants, but first we need to show the counterpart of Lemma 3.2, Corollary 3.3, and Lemma 3.4 for $d$-weighted Fibonacci numbers. 
Lemma 4.2. For any $\gamma \in\left\langle 0, f_{n-1}^{d}\right\rangle$, there exist $\beta, \alpha_{0}, \ldots, \alpha_{n-3} \in\langle 0, d\rangle$ such that

$$
\gamma=\beta+\sum_{i=0}^{n-3} \alpha_{i} f_{i}^{d}
$$

where the sequence $\left(f_{i}^{d}\right)_{i \in \mathbb{N}}$ is defined in (4.9).

Proof. The proof is by induction on $n$. The base cases $n=1$ and $n=2$ are trivially satisfied.

For the inductive step, if $\gamma \leq f_{n-1}^{d}$, then the statement is true by the inductive hypothesis. Otherwise, we have that $f_{n-1}^{d}<\gamma \leq f_{n}^{d}$, which implies that $\gamma-d f_{n-2}^{d} \leq f_{n-1}^{d}$. Therefore, there exist $\beta^{\prime}, \alpha_{0}^{\prime}, \ldots, \alpha_{n-3}^{\prime}$ in $\langle 0, d\rangle$ such that

$$
\gamma-d f_{n-2}^{d}=\beta^{\prime}+\sum_{i=0}^{n-3} \alpha_{i}^{\prime} f_{i}^{d}
$$

It follows that

$$
\gamma=\beta^{\prime}+\sum_{i=0}^{n-3} \alpha_{i}^{\prime} f_{i}^{d}+d f_{n-2}^{d}=\beta+\sum_{i=0}^{n-2} \alpha_{i} f_{i}^{d}
$$

where $\beta=\beta^{\prime}, \alpha_{i}=\alpha_{i}^{\prime}$ for $i=0, \ldots, n-3$, and $\alpha_{n-2}=d$.

Lemma 4.2 can be related to a famous and perhaps surprising result for standard Fibonacci numbers, known as Zeckendorf's theorem [1], which we now state.

THEOREM 4.3. (Zeckendorf's theorem) Every nonzero natural number $m \in \mathbb{N}$ can be written as the sum of one or more distinct Fibonacci numbers so that the sum does not contain two consecutive Fibonacci numbers, i.e.,

$$
m=\sum_{i}^{k} f_{c_{i}}
$$

where $c_{i} \geq 2$ and $c_{i+1}>c_{i}+1$. Moreover, this representation is unique.

Corollary 4.4. For any $\gamma \in\left\langle-f_{n-1}^{d}, f_{n-1}^{d}\right\rangle$, there exist $\beta, \alpha_{0}, \ldots, \alpha_{n-3} \in\langle-d, d\rangle$ such that

$$
\gamma=\beta+\sum_{i=0}^{n-3} \alpha_{i} f_{i}^{d}
$$

where the sequence $\left(f_{i}^{d}\right)_{i \in \mathbb{N}}$ is defined in (4.9).

Proof. If $\gamma \geq 0$, then (4.15) follows from Lemma 4.2. For $\gamma<0$, the coefficients in (4.15) can be obtained by changing the sign of those in the representation of $-\gamma$ in (4.13).

LEMMA 4.5. If $d>1$, for any $\gamma \in\left\langle-f_{n-1}^{d}, f_{n-1}^{d}\right\rangle$, there exist $\beta, \alpha_{0}, \ldots, \alpha_{n-3} \in\langle-d, d\rangle^{\circ}$ such that

$$
\gamma=\beta+\sum_{i=0}^{n-3} \alpha_{i} f_{i}^{d}
$$

where the sequence $\left(f_{i}^{d}\right)_{i \in \mathbb{N}}$ is defined in (4.9). 


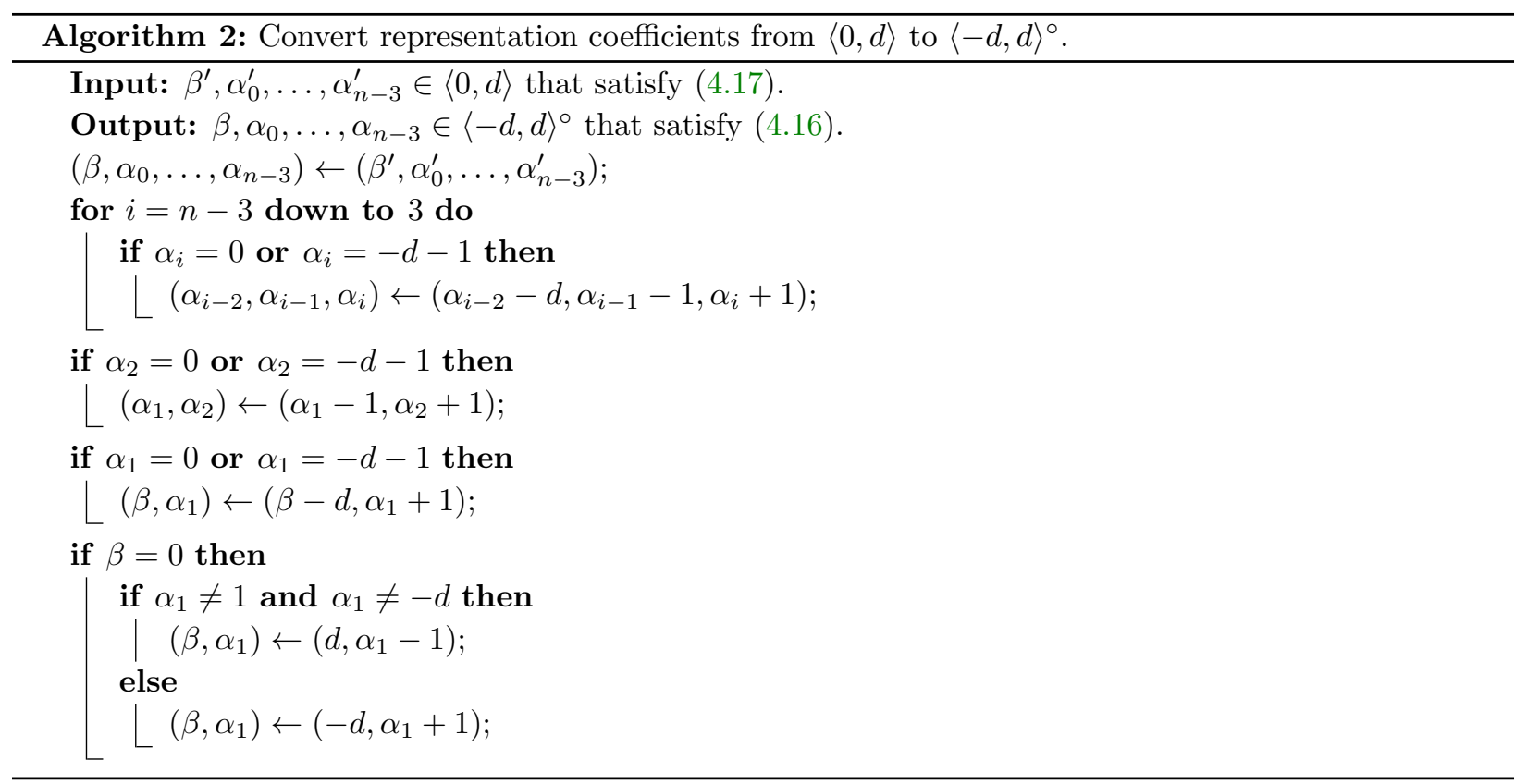

Proof. From Lemma 4.2, if $\gamma \geq 0$ then there exist $\beta^{\prime}, \alpha_{0}^{\prime}, \ldots, \alpha_{n-3}^{\prime}$ in $\langle 0, d\rangle$ such that

$$
\gamma=\beta^{\prime}+\sum_{i=0}^{n-3} \alpha_{i}^{\prime} f_{i}^{d}
$$

The pseudocode in Algorithm 2 shows that the coefficients in the representation (4.16) can be computed from those of (4.17). The case $\gamma<0$ is analogous, it suffices to consider the representation in (4.17) for $-\gamma$ and change the sign of the coefficients thus obtained.

We need one last technical lemma. In Proposition 3.6, we use a result by Ching [4] to count the number of nonzero terms in the determinant expression of a Hessenberg matrix. Lemma 4.6 achieves the same goal for matrices in $\mathcal{H}_{0}^{n}(\mathcal{D})$.

LEMMA 4.6. Let $H \in \mathcal{H}_{0}^{n}(\mathcal{D})$, let $p_{n}\left(h_{12}, \ldots, h_{(n-1) n}\right)$ be the determinant of $H$ seen as a polynomial in the $n(n-1) / 2$ variables $h_{12}, \ldots, h_{(n-1) n}$, and let $\mathbf{a}_{j}$ denote a monomial of $p_{n}$. Then

$$
p_{n}\left(h_{12}, \ldots, h_{(n-1) n}\right)=(-1)^{n} \sum_{j=0}^{f_{n-1}}(-1)^{\operatorname{deg} \mathbf{a}_{j}} \mathbf{a}_{j}
$$

where $f_{n}$ is the nth Fibonacci number and $\operatorname{deg} \mathbf{a}_{j}$ is the total degree of the monomial $\mathbf{a}_{j}$. 
Proof. We will prove this claim by induction on the matrix

$$
H=\left[\begin{array}{ccccc}
0 & h_{12} & h_{13} & \cdots & h_{1 n} \\
1 & 0 & h_{23} & \cdots & h_{2 n} \\
& 1 & \ddots & \ddots & \vdots \\
& & \ddots & 0 & h_{(n-1) n} \\
& & & 1 & 0
\end{array}\right]
$$

The claim is true for the base cases $n=1, n=2$, and $n=3$, since $p_{1}(0)=0$,

$$
p_{2}\left(h_{12}\right)=-h_{12}=(-1)^{2} \cdot(-1) \cdot h_{12},
$$

and

$$
p_{3}\left(h_{12}, h_{13}, h_{23}\right)=h_{13}=(-1)^{3} \cdot(-1) \cdot h_{13} .
$$

Then, by using the Laplace expansion twice, we get

$$
\operatorname{det} H_{n}=-h_{12}\left|\begin{array}{cccccc}
0 & h_{34} & h_{35} & h_{36} & \cdots & h_{3 n} \\
1 & 0 & h_{45} & h_{46} & \cdots & h_{4 n} \\
& 1 & 0 & h_{56} & \cdots & h_{5 n} \\
& & 1 & \ddots & \ddots & \vdots \\
& & & \ddots & 0 & h_{(n-1) n} \\
& & & & 1 & 0
\end{array}\right|-\left|\begin{array}{cccccc}
0 & h_{13} & h_{14} & h_{15} & \cdots & h_{1 n} \\
1 & 0 & 0 & 0 & \cdots & 0 \\
& 1 & 0 & h_{45} & \cdots & h_{4 n} \\
& & \ddots & \ddots & \ddots & \vdots \\
& & & 0 & h_{(n-1) n} \\
& & & & 1 & 0
\end{array}\right|
$$

which reads, using the inductive hypothesis,

$$
\begin{aligned}
& p_{n}\left(h_{12}, \ldots, h_{(n-1) n}\right)=-h_{12} p_{n-2}\left(h_{34}, \ldots, h_{(n-1) n}\right)-p_{n-1}\left(h_{13}, \ldots, h_{(n-1) n}\right) \\
& =h_{12}(-1)^{n-1} \sum_{k=0}^{f_{n-3}}(-1)^{\operatorname{deg} \widehat{\mathbf{a}}_{k}} \widehat{\mathbf{a}}_{k}+(-1)^{n} \sum_{j=0}^{f_{n-2}}(-1)^{\operatorname{deg} \mathbf{a}_{j}} \mathbf{a}_{j} \\
& =(-1)^{n} \sum_{k=0}^{f_{n-3}}(-1)^{\operatorname{deg} \mathbf{a}_{k}} \mathbf{a}_{k}+(-1)^{n} \sum_{j=0}^{f_{n-2}}(-1)^{\operatorname{deg} \mathbf{a}_{j}} \mathbf{a}_{j},
\end{aligned}
$$

where $h_{12} \widehat{\mathbf{a}}_{k}=\mathbf{a}_{k}$. This proves the claim about the sign of the monomials.

For the number of terms in the formula, note that the total number of monomials in $p_{n}\left(h_{12}, \ldots, h_{(n-1) n}\right)$ cannot exceed $f_{n-2}+f_{n-3}=f_{n-1}$, but could be smaller as some monomials might cancel out. However, these cancellations cannot occur, because every monomial in $p_{n-2}\left(h_{34}, \ldots, h_{(n-1) n}\right)$ contains one coefficient among $h_{34}, h_{35}, \ldots, h_{3 n}$ that does not appear in $p_{n-1}\left(h_{13}, \ldots, h_{(n-1) n}\right)$. This concludes the proof. 
Proposition 4.7. If $d>1$, then the set of possible determinants of matrices in the family $\mathcal{H}_{0}^{n}\left(\langle-d, d\rangle^{\circ}\right)$ is $\left\langle-f_{n-1}^{d}, f_{n-1}^{d}\right\rangle$, where $f_{n}^{d}$ is the $n$th $d$-weighted Fibonacci number as defined in (4.9).

Proof. In this case, we show how to construct a matrix $H \in \mathcal{H}_{0}^{n}\left(\langle-d, d\rangle^{\circ}\right)$ such that $\operatorname{det} H=k$ for any $k \in\left\langle-f_{n-1}^{d}, f_{n-1}^{d}\right\rangle$. Let

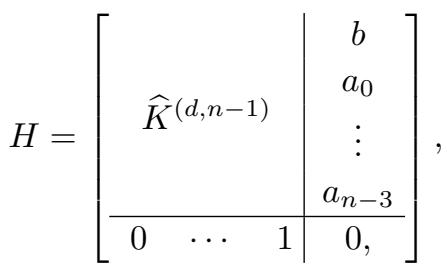

where $\widehat{K}^{(d, n-1)}$ is defined in (4.11). By Lemma 2.1 and Proposition 4.1, it follows that

$$
\begin{aligned}
\operatorname{det} H & =(-1)^{n+1} b+\sum_{i=0}^{n-3}(-1)^{n+i} a_{i} \operatorname{det} \widehat{K}^{(d, i+1)} \\
& =\widetilde{b}+\sum_{i=0}^{n-3} \widetilde{a}_{i} f_{i}^{d},
\end{aligned}
$$

where $\widetilde{b}=(-1)^{n+1} b$ and $\widetilde{a}_{i}=(-1)^{n+1} a_{i}$, for $i=0, \ldots, n-3$. Since $b, a_{0}, \ldots, a_{n-3} \in\langle-d, d\rangle^{\circ}$, by Lemma 4.5 we can conclude that the last column of $H$ can be chosen so that $\operatorname{det} H=k$ for any $k \in\left\langle-f_{n-1}^{d}, f_{n-1}^{d}\right\rangle$.

Proposition 4.8. The set of possible determinants of matrices in the family $\mathcal{H}_{0}^{n}\left(\langle-1,1\rangle^{\circ}\right)$ is

$$
\left\{-f_{n-1},-f_{n-1}+2,-f_{n-1}+4, \ldots, f_{n-1}-4, f_{n-1}-2, f_{n-1}\right\}
$$

where $f_{n}$ is the nth Fibonacci number as defined in (4.9).

Proof. By Lemma 4.6, there are only $f_{n-1}$ possibly nonzero terms in the determinant expression of a $n \times n$ Hessenberg matrix with a zero diagonal. If a matrix is in $\mathcal{H}_{0}^{n}\left(\langle-1,1\rangle^{\circ}\right)$, then each of these $f_{n-1}$ monomials evaluates to either +1 or -1 , which implies that the determinant of any such matrices must have the same parity as $f_{n-1}$. Now we explain how to build a matrix $H \in \mathcal{H}_{0}^{n}\left(\langle-1,1\rangle^{\circ}\right)$ such that $\operatorname{det} H=f_{n-1}-2 k$ for $k \in\left\langle 0, f_{n-1}\right\rangle$. Let

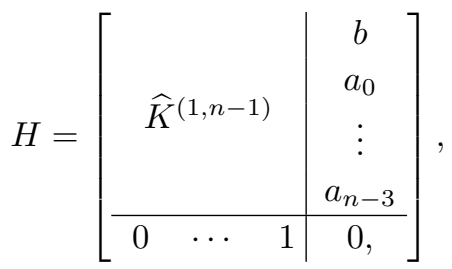

where $\widehat{K}^{(1, n-1)}$ is defined in (4.11). By Lemma 2.1 and Proposition 4.1, we have that

$$
\begin{aligned}
\operatorname{det} H & =(-1)^{n+1} b+\sum_{i=0}^{n-3}(-1)^{n+i} a_{i} \operatorname{det} \widehat{K}^{(1, i+1)} \\
& =\widetilde{b}+\sum_{i=0}^{n-3} \widetilde{a}_{i} f_{i},
\end{aligned}
$$

where $\widetilde{b}=(-1)^{n+1} b$ and $\widetilde{a}_{i}=(-1)^{n+1} a_{i}$, for $i=0, \ldots, n-3$. By Lemma 4.2 , we can choose $\widetilde{b}_{\text {and }} \widetilde{a}_{i}$ in $\{0,1\}$ so that $\widetilde{b}+\sum_{i=0}^{n-3} \widetilde{a}_{i} f_{i}=k$ for $k \in\left\langle 0, f_{n-1}\right\rangle$. If we then substitute the coefficients that are zeros with 
-1 , then the last column is such that

$$
\operatorname{det} H=\widetilde{b}+\sum_{i=0}^{n-3} \widetilde{a}_{i} f_{i}=f_{n-1}-2 k
$$

which concludes the proof.

Proposition 4.9. For any $d \in \mathbb{N}$, the set of possible determinants of matrices in the family $\mathcal{H}_{0}^{n}(\langle-d, d\rangle)$ is $\left\langle-f_{n-1}^{d}, f_{n-1}^{d}\right\rangle$, where $f_{n}$ is the nth Fibonacci number as defined in (4.9).

Proof. The proof is analogous to that of Proposition 4.7: it suffices to use Corollary 4.4 in lieu of Lemma 4.5.

Proposition 4.10. For any $d \in \mathbb{N}$, the set of possible determinants of matrices in the family $\mathcal{H}_{0}^{n}(\langle-d, 0\rangle)$ is $(-1)^{n} \cdot\left\langle 0, f_{n-1}^{d}\right\rangle$, where $f_{n}$ is the nth Fibonacci number as defined in (4.9).

Proof. This proof follows the lines of the proof of Proposition 4.7. Note that $\mathcal{H}_{0}^{n}(\langle-d, 0\rangle) \subset \mathcal{H}_{0}^{n}(\langle-d, d\rangle)$, and that since $\widehat{K}^{(d, n-1)} \in \mathcal{H}_{0}^{n}(\langle-d, 0\rangle)$, the matrix $H$ in $(4.19)$ is in $\mathcal{H}_{0}^{n}(\langle-d, 0\rangle)$ if $b, a_{0}, \ldots, a_{n-3} \in\langle-d, 0\rangle$. Combining Lemma 2.1 and Proposition 4.1 in this case gives

$$
\begin{aligned}
\operatorname{det} H & =(-1)^{n+1} b+\sum_{i=0}^{n-3}(-1)^{n+i} a_{i} \operatorname{det} \widehat{K}^{(d, i+1)} \\
& =(-1)^{n}\left(\tilde{b}+\sum_{i=0}^{n-3} \widetilde{a}_{i} f_{i}^{d}\right)
\end{aligned}
$$

where $\widetilde{b}=-b$ and $\widetilde{a}_{i}=-a_{i}$, for $i=0, \ldots, n-3$. Since $\widetilde{b}, \widetilde{a}_{0}, \ldots, \widetilde{a}_{n-2} \in\langle 0, d\rangle$, by Lemma 4.2 , we can choose the last column of $H$ so that $\operatorname{det} H=(-1)^{n} \cdot k$ for all $k \in\left\langle 0, f_{n-1}^{d}\right\rangle$.

Propositions 4.10, 4.9, and 4.8 show [13, Conjecture 12], [13, Conjecture 14], and [13, Conjecture 16], respectively. For $d=1$, these results show that the number of distinct determinants of normalized hollow Bohemian upper Hessenberg matrices of size $n$ with entries from the sets $\langle-1,0\rangle$ and $\langle-1,1\rangle^{\circ}$ is given by the $n$th element of the OEIS sequence A001611. For the family of matrices with elements drawn from $\langle-1,1\rangle$, an analogous result holds for the OEIS sequence A001588.

5. Future developments. In the previous sections, we generalized all conjectures in [13] dealing with upper Hessenberg matrices, except [13, Conjecture 8] and [13, Conjecture 9]. The latter has so far resisted our efforts, and will be the subject of future investigation. In this section, we show that the former is true and state a generalization we were unable to prove. We will make use of the numbers defined by the recursion

$$
\left\{\begin{array}{l}
g_{1}^{d}=d, \\
g_{2}^{d}=d^{2}, \\
g_{n}^{d}=d g_{n-1}^{d}+g_{n-2}^{d}, \quad n \in \mathbb{N} \backslash\{0,1,2\} .
\end{array}\right.
$$

The sequence in (5.20) is a generalization of Fibonacci numbers which grows significantly faster than that in (4.9). 
Conjecture 5.1. We have that

$$
\max _{H \in \mathcal{H}^{n}(\langle 0, d\rangle)}|\operatorname{det} H|=\operatorname{det} L^{(d, n)}=g_{n}^{d},
$$

where $g_{n}$ is defined in (5.20) and

$$
\ell_{i j}^{(d, n)}=\left\{\begin{array}{l}
0, \quad i>j+1 \text { or } i<j \text { and } i+j \text { is odd } \\
1, \quad i=j+1 \\
d, \quad i \leq j \text { and } i+j \text { is even. }
\end{array}\right.
$$

Evidence of this is the fact that the entries in the upper triangular part of a matrix with largest absolute determinant must be either 0 or $d$. This can be easily seen by noting that the determinant is just the signed sum of the product of entries of $L^{(d, n)}$. The proof for $d=1$ is a special case of [4, Theorem 1]. In the following we supply a proof for the case $d=2$.

Proof of Conjecture 5.1 for $d=2$. The second equality in (5.21) can be proven easily by induction, by expanding along the last row of $L^{(2, n)}$ and noting that $\ell_{n n}^{(2, n)}=d$. The first equality can also be proven by induction. It is straightforward to check that the result holds for $n=1,2$, and 3 . For $n \geq 4$, let $H \in \mathcal{H}^{n}(\langle 0,2\rangle)$. By expanding along the first column of $H$ we have

$$
\operatorname{det} H=h_{11}\left|\begin{array}{cccc}
h_{22} & h_{23} & \cdots & h_{2 n} \\
1 & h_{33} & \cdots & h_{3 n} \\
& \ddots & \ddots & \vdots \\
& & 1 & h_{n n}
\end{array}\right|-\left|\begin{array}{cccc}
h_{12} & h_{13} & \cdots & h_{1 n} \\
1 & h_{33} & \cdots & h_{3 n} \\
& \ddots & \ddots & \vdots \\
& & 1 & h_{n n}
\end{array}\right| .
$$

If $h_{11} \neq 2$ or $h_{12}=0$, then $|\operatorname{det} H| \leq 2 g_{n-1}^{2}+g_{n-2}^{2}=g_{n}^{2}$, and the proof is completed. Otherwise, we can expand along the first column of the two resulting matrices, to obtain

$$
\operatorname{det} H=\left(2 h_{22}-h_{12}\right)\left|\begin{array}{cccc}
h_{33} & h_{34} & \cdots & h_{3 n} \\
1 & h_{44} & \cdots & h_{4 n} \\
& \ddots & \ddots & \vdots \\
& & 1 & h_{n n}
\end{array}\right|-2\left|\begin{array}{cccc}
h_{23} & h_{24} & \cdots & h_{2 n} \\
1 & h_{44} & \cdots & h_{4 n} \\
& \ddots & \ddots & \vdots \\
& & 1 & h_{n n}
\end{array}\right|+\left|\begin{array}{cccc}
h_{13} & h_{14} & \cdots & h_{1 n} \\
1 & h_{44} & \cdots & h_{4 n} \\
& \ddots & \ddots & \vdots \\
& & 1 & h_{n n}
\end{array}\right| .
$$

If $h_{13}=0$, then $|\operatorname{det} H| \leq 5 g_{n-2}^{2}+g_{n-3}^{2}<g_{n}^{2}$, which as above would conclude the proof. Otherwise, the last two determinants can be rewritten, by expanding along the last row and collecting like terms, as

$$
\left(h_{13}-2 h_{23}\right)\left|\begin{array}{cccc}
h_{44} & h_{45} & \cdots & h_{4 n} \\
1 & h_{55} & \cdots & h_{5 n} \\
& \ddots & \ddots & \vdots \\
& & 1 & h_{n n}
\end{array}\right|+2\left|\begin{array}{cccc}
h_{24} & h_{25} & \cdots & h_{2 n} \\
1 & h_{55} & \cdots & h_{5 n} \\
& \ddots & \ddots & \vdots \\
& & 1 & h_{n n}
\end{array}\right|-\left|\begin{array}{cccc}
h_{14} & h_{15} & \cdots & h_{1 n} \\
1 & h_{55} & \cdots & h_{5 n} \\
& \ddots & \ddots & \vdots \\
& & 1 & h_{n n}
\end{array}\right|,
$$

and substituting (5.24) into (5.23) yields

$$
|\operatorname{det} H| \leq\left|2 h_{22}-h_{12}\right| g_{n-2}^{2}+\left|h_{13}-2 h_{23}\right| g_{n-3}^{2}+3 g_{n-3}^{2} \leq 3 g_{n-2}^{2}+6 g_{n-3}^{2}<g_{n}^{2},
$$

which concludes the proof.

This proof does not generalize to $d>2$, but shows that [13, Conjecture 8] is true, since the maximum absolute determinant of matrices in $\mathcal{H}_{0}^{n}(\langle 0,2\rangle)$ is the $n$th element of the OEIS sequence A052542. 
Acknowledgments. We are grateful to Nicholas J. Higham for his feedback on early drafts of this manuscript. We thank the anonymous referee for his comments and in particular for pointing us to a theorem by Gibson [8] that can be used to prove some of our results in an alternative way.

\section{REFERENCES}

[1] J.L. Brown. Zeckendorf's theorem and some applications. Fibonacci Quart., 2(3):163-168, 1964.

[2] E.Y.S. Chan, R.M. Corless, L. González-Vega, J.R. Sendra, J. Sendra, and S.E. Thornton. Bohemian upper Hessenberg matrices. Preprint, arXiv:1809.10653, 2018.

[3] E.Y.S. Chan, R.M. Corless, L. González-Vega, J.R. Sendra, J. Sendra, and S.E. Thornton. Bohemian upper Hessenberg Toeplitz matrices. Preprint, arXiv:1809.10664, 2018.

[4] L. Ching. The maximum determinant of an $n \times n$ lower Hessenberg $(0,1)$ matrix. Linear Algebra Appl., 183:147-153, 1993.

[5] R.M. Corless and S.E. Thornton. The Bohemian eigenvalue project. ACM Commun. Comput. Algebra, 50(4):158-160, 2017.

[6] J.N. Franklin. Matrix Theory. Prentice-Hall, Englewood Cliffs, NJ, USA, 1968.

[7] C.W. Gear. A simple set of test matrices for eigenvalue programs. Math. Comp., 23(105):119-119, 1969.

[8] P.M. Gibson. An identity between permanents and determinants. Amer. Math. Monthly, 76:270-271, 1969.

[9] J.S. Hadamard. Résolution d'une question relative aux determinants. Bulletin des Sciences Mathematiques, 17:240-246, 1893.

[10] J.J. Sylvester. LX. Thoughts on inverse orthogonal matrices, simultaneous signsuccessions, and tessellated pavements in two or more colours, with applications to Newton's rule, ornamental tile-work, and the theory of numbers. The London, Edinburgh, and Dublin Philosophical Magazine and Journal of Science, Series 4, 34(232):461-475, 1867.

[11] O. Taussky. Matrices of rational integers. Bull. Amer. Math. Soc., 66(5):327-346, 1960.

[12] O. Taussky. Some computational problems involving integral matrices. Journal of Research of the National Bureau of Standards - B. Mathematics and Mathematical Physics, 65(1):15-17, 1961.

[13] S.E. Thornton. The characteristic polynomial database. Available at http://bohemianmatrices.com/cpdb, 2018. 